\title{
Single-letter recognition accuracy benefits and position information
}

\author{
A. H. C. VAN DER HEIJDEN, G. WOLTERS, E. FLEUR, and J. G. M. HOMMELS \\ Leiden University, Leiden, The Netherlands \\ (Charles W. Eriksen, Sponsor)
}

\begin{abstract}
Beneficial effects of location cuing in single-letter recognition tasks have been found in a number of studies. Van der Heijden, Neerincx, and Wolters (1989) have argued that such identity benefits can be expressed as a fraction of the room for improvement in position information. Their model suggests that an attentional process may, through the addition of location information, enhance identification performance. For this model to be correct, however, two conditions must be met: (1) position information of the target letters has to be missing in a proportion of the trials, and (2) identity benefits have to be smaller than the room for improvement in position information. Two experiments are reported in which position and identity information of single target letters are determined under identical conditions. The outcomes of both experiments are consistent with the conditions suggested by the model.
\end{abstract}

Demonstrating positive selective attention effects on recognition accuracy with single-item displays has proved very hard. Grindley and Townsend (1968) tried but failed. In subsequent location-cuing experiments, attentional benefits were demonstrated, but the effects were generally very small (from $2 \%$ to $5 \%$; see, e.g., Egly \& Homa, 1984; van der Heijden et al., 1989; van der Heijden, Schreuder, \& Wolters, 1985; van der Heijden, Wolters, Groep, \& Hagenaar, 1987).

Van der Heijden et al. (1987) have tried to answer the question of why benefits are so difficult to find. They argue that the main reason has to be found in the fact that in these experiments a performance baseline, $I_{\mathrm{nc}}$, obtained in a nocue condition is used. So, in the cue condition, no improvement in recognition accuracy is possible on a proportion $I_{\mathrm{nc}}$ of the trials, leaving only a relatively small "room for improvement" in a proportion $\left(1-I_{\mathrm{nc}}\right)$ of the trials. Analysis of a great number of experiments suggested that $\left(1-I_{\mathbf{n c}}\right)$ is indeed the prime limiting factor (see, e.g., van der Heijden et al., 1989; van der Heijden et al., 1987). It was invariantly found that the subject's benefits, $\Delta I$, were related to their rooms for improvement $\left(1-I_{\mathrm{nc}}\right)$, according to:

$$
\Delta I=a\left(1-I_{\mathrm{nc}}\right)-b .
$$

Van der Heijden et al. (1989), however, have pointed out that, as a model for cuing, Equation 1 has serious drawbacks. The equation simply gives an empirical relation and does not allow an interpretation that explains why location cuing enhances identification performance. There-

Correspondence should be addressed to A. H. C. van der Heijden, Leiden University, Unit of Experimental and Theoretical Psychology, Wassenaarseweg 52, 2333 AK Leiden, The Netherlands. fore, van der Heijden et al. (1989) have derived an alternative for Equation 1 on the basis of evidence reported by Egly and Homa (1984).

Egly and Homa (1984) studied single-item letter and position naming. In the first task, the identity, and in the second task, the position of a peripheral letter had to be named. One important observation was that in the positionnaming task subjects made a lot of errors. Moreover, benefits brought by (approximate) position cuing were larger in the position-naming task than in the letter-naming task. This suggested the possibility that identity benefits, $\Delta I$, are a fraction of the room for improvement, not in terms of identity information $\left(1-I_{n c}\right)$, but in terms of position information $\left(1-P_{n c}\right)$ :

$$
\Delta I=g\left(1-P_{\mathrm{nc}}\right) \text {. }
$$

In a theoretical analysis, it could be shown that Equation 2 is consistent with Equation 1 and with the available experimental data (see van der Heijden et al., 1989, and van der Heijden, 1991). Moreover, Equation 2 provides a first step from an uninterpretable empirical relation to an interpretable model. According to that model, there are two reasons for errors in a single-item identification task. First, even when the location of the stimulus is known perfectly, identification errors sometimes occur. Second, when there are no locational cues, on some proportion of the trials the subject does not know where in the display the item has appeared. Then the subject does not know which of the possible item locations should be the source of the identification decision. The advantage provided by the locational cue is that it eliminates the second source of error. So, basically, the model suggested by Equation 2 states that central selective attention in single-item recognition tasks is effective in enhancing identification performance because cuing eliminates location uncertainty. 
As indicated, however, at the basis of Equation 2 is accidental evidence provided by Egly and Homa (1984). To assess the viability of Equation 2 as a model, exact evidence on position information and identity information under identical exposure conditions is needed. More specifically, the knowledge needed is, whether, with the exposure conditions used in single-item recognition tasks, on a proportion of trials position information is missing and the room for improvement in position information is larger than the accuracy benefit (i.e., whether $\Delta I$ can indeed be expressed as a fraction of $1-P_{n c}$ ).

In this study, two experiments are reported that investigate the two issues mentioned above. In Experiment 1, the letters were presented just as in our previous experiments. In Experiment 2, the onset of the stimulus letter was degraded by presenting masks of which the disappearance coincided with the start of the target letter.

\section{EXPERIMENT 1}

\section{Method}

Subjects. Six students of the University of Leiden served as paid subjects. All had normal or corrected-to-normal vision.

Apparatus. The stimuli were presented on a fast display screen (Vector General VR-17). Stimulus presentation and response registration were controlled by a PDP-11/34 computer.

Stimuli. The letters used were E, F, H, M, O, Q, K, and X. Each letter subtended maximal visual angles of $.28^{\circ}$ (width) and $.34^{\circ}$ (height) at a viewing distance of $98 \mathrm{~cm}$. Stimuli were presented in one out of five equally spaced positions on the circumference of an imaginary circle around the fixation point (a dim asterisk). The circle had a radius of $2^{\circ}$. One of the positions was at 12 o'clock. Low-intensity dots were used as position indicators. The dots were lying on the circumference of an imaginary circle with a radius of $2.6^{\circ}$. In the cue condition, a single dot was presented at the position corresponding with the position of the impending target letter. In the no-cue condition, dots were presented simultaneously at all five possible letter positions.

Design. A within-subject design with repeated measures was used. Subjects received blocks of $\mathbf{4 0}$ trials. Each block consisted entirely of either cued or uncued trials in combination with one of the three experimental tasks: letter naming, position naming, or letter-plus-position naming. Within each block, each letter was presented once in each position. Order of letters and positions within a block was random. The order of blocks (i.e., cuing condition and task combinations) was systematically varied over days and subjects by means of a Latin square.

Procedure. Subjects participated on 7 consecutive working days. They were run individually in a dimly illuminated room. The 1 st day was used for practice only. During this practice session, the $60 \%$ correct letter recognition threshold was determined by varying the presentation times of the letters in a position-cued condition. The presentation durations ranged from 10 to $28 \mathrm{msec}$. On the 6 experimental days, testing started with two practice blocks, followed by six experimental blocks. Before each block, the subjects were informed about the task that they had to perform. In the letter-naming task, the identity of the letter had to be given. In the position-naming task, the position of the letter had to be given. In the letter- plus position-naming task, both letter identity and letter position had to be indicated. In all tasks, subjects had to guess if necessary.

The presentation sequence per trial consisted of a cue exposure (either one or five dots) for $25 \mathrm{msec}$, immediately followed by the presentation of a stimulus letter for the predetermined duration. The next trial was initiated by the subject by pressing a button built in the table surface in front of him/her.

\section{Results and Discussion}

Per subject, day, task and cue condition, the number of correct responses was determined. Each of these scores is based on one block of 40 trials. Percentages correct,
Table 1

Mean Percentage Correct per Subject, per Task, and per Cuing Condition

\begin{tabular}{|c|c|c|c|c|c|c|}
\hline \multirow[b]{2}{*}{ Subjects } & \multicolumn{2}{|c|}{ Letter } & \multicolumn{2}{|c|}{ Position } & \multicolumn{2}{|c|}{$\begin{array}{c}\text { Letter and } \\
\text { Position }\end{array}$} \\
\hline & Cue & No Cue & Cue & No Cue & Cue & No Cue \\
\hline 1 & 65.8 & 60.0 & 100.0 & 98.7 & 62.9 & 60.4 \\
\hline 2 & 69.6 & 63.7 & 100.0 & 90.8 & 73.3 & 65.8 \\
\hline 3 & 72.9 & 72.9 & 100.0 & 98.7 & 72.5 & 67.9 \\
\hline 4 & 64.6 & 60.8 & 100.0 & 98.3 & 64.2 & 62.1 \\
\hline 5 & 61.2 & 58.7 & 100.0 & 92.9 & 64.6 & 57.1 \\
\hline 6 & 62.1 & 60.4 & 100.0 & 92.9 & 53.3 & 50.8 \\
\hline$M$ & 66.0 & 62.8 & 100.0 & 95.4 & 65.1 & 60.7 \\
\hline
\end{tabular}

averaged over days, are presented in Table 1 . The three experimental tasks were analyzed separately.

Letter naming. An analysis of variance (ANOVA) showed a significant effect of cuing $[F(1,5)=11.7, p<$ $.05]$. Average proportions correct were .628 in the nocue condition, and .660 in the cue condition. The effect of days was not significant $[F(5,25)=1.3]$.

Position naming. Analysis revealed a significant cuing effect $[F(1,5)=9.8, p<.05]$. The average proportion correct in the no-cue condition was .954 , whereas position naming was perfect in the cue condition. The effect of days was not significant $[F(5,25)=.7]$.

Position and letter naming. Also here a significant effect of cuing was found $[F(1,5)=18.6, p<.01]$. Average proportions correct in the no-cue and cue conditions were .607 and .651 . The effect of days was not significant $[F(5,25)=1.4]$. This task can also be scored as a letternaming task and as a position-naming task. When scored as a letter-naming task, the average proportion correct in the no-cue condition was .620 and in the cue condition .654 . When scored as a position-naming task, the average proportion correct in the no-cue condition equaled .944 and in the cue condition .997 .

From these data, it will be clear that the two conditions that we specified in the introduction, and that are required for our tentative model for identity benefits brought by cuing, are satisfied. First, on a proportion of no-cue trials, position information is lacking. On the average, this proportion is .046 in the position-naming task and .056 in the position- and letter-naming task. So in this type of task, there is indeed room for improvement in position information. Second, this room for improvement in position information is indeed larger than the identity benefit brought by cuing, which on the average is .032 in the letter-naming task and .034 in the position- and letter-naming task.

\section{EXPERIMENT 2}

An important observation in Experiment 1 was that in the no-cue condition, subjects sometimes did not know where the target letter was located. However, localization performance in the position-naming task and in the positionand letter-naming task was rather high. Experiment 2 was set up to decrease the location information provided by the letter itself. 
Position information provided by the target letter was decreased by "hiding" the onset of the letter. Target-letter presentation was preceded by small masks on all possible letter positions. Because the offset of the masks coincided with the onset of the target letter, it is likely that the target letter itself does not act as a unique and abrupt location cue (see, e.g., Yantis \& Jonides, 1984). Because in Experiment 1 position and letter naming gave results comparable to those of the position-naming and letter-naming tasks separately, only one task was used: subjects had to respond by naming both the letter and the position.

\section{Method}

Subjects. Eight students of the University of Leiden participated as subjects on 5 consecutive days.

Apparatus and Stimuli. The display and the letters were the same as in Experiment 1 . The letters subtended a visual angle of $.28^{\circ} \times .34^{\circ}$ at a viewing distance of $98 \mathrm{~cm}$. At the beginning of each trial, five masks were presented at equally spaced positions on the circumference of an imaginary circle with a radius of $1.7^{\circ}$ around the fixation point. One of the positions was at $12 \mathrm{o}$ 'clock. The masks consisted of small squares $\left(.34^{\circ} \times .34^{\circ}\right.$ of visual angle) composed of small vertical lines. To the observer, they looked like evenly illuminated squares. As a cue, a pair of dots was presented on corresponding positions on the circumference of two similar imaginary circles with radii of $2.0^{\circ}$ and $1.4^{\circ}$. So the dots appeared slightly at the outside and the inside of the mask. In the nocue condition, pairs of dots were presented at all five positions.

Desigen. Stimuli were presented in blocks of 80 trials. Within a block, each of the eight target letters was presented twice, once in the cue and once in the no-cue condition, at each of the five possible locations. The order of letters, positions, and cuing conditions within a block was randomized.

Procedure. Subjects were run individually in a dimly illuminated room. The 1st day was used for determining the stimulus presentation duration resulting in $75 \%$ correct recognition of letters in a no-cue condition. The presentation durations ranged from 20 to $90 \mathrm{msec}$. On the next 4 experimental days, subjects started with three practice blocks, followed by four experimental blocks. Each trial was started by the subject by pressing a button. After a $200-\mathrm{msec}$ delay, the five masks appeared for $800 \mathrm{msec}$. During the last $50 \mathrm{msec}$ of the presentation of the masks, either one or five cues were shown. Immediately after the termination of the masks and the cues, a letter was presented at one of the five mask positions for the predetermined duration. Subjects had to respond by naming both the letter and its position.

\section{Results and Discussion}

From the raw data, correct letter identification scores, position scores, and letter-plus-position scores were de-

Table 2

Mean Percentages Correct per Subject and per Cuing Condition for the Letter- and Position-Naming Task

\begin{tabular}{|c|c|c|c|c|c|c|}
\hline \multirow[b]{2}{*}{ Subjects } & \multicolumn{2}{|c|}{ Letter } & \multicolumn{2}{|c|}{ Position } & \multicolumn{2}{|c|}{$\begin{array}{l}\text { Letter and } \\
\text { Position }\end{array}$} \\
\hline & Cue & No Cue & Cue & $\overline{\text { No Cue }}$ & Cue & No Cue \\
\hline 1 & 65.0 & 63.9 & 99.5 & 88.8 & 64.8 & 62.3 \\
\hline 2 & 75.2 & 73.4 & 99.2 & 89.7 & 74.8 & 72.3 \\
\hline 3 & 73.4 & 73.0 & 100.0 & 96.1 & 73.4 & 71.9 \\
\hline 4 & 73.8 & 70.8 & 99.8 & 89.5 & 73.8 & 70.0 \\
\hline 5 & 75.5 & 71.1 & 99.7 & 92.8 & 75.5 & 70.4 \\
\hline 6 & 77.2 & 74.2 & 99.8 & 93.3 & 77.0 & 72.7 \\
\hline 7 & 72.8 & 70.8 & 99.8 & 88.8 & 72.8 & 68.3 \\
\hline 8 & 72.7 & 74.2 & 99.7 & 94.1 & 72.5 & 72.8 \\
\hline$M$ & 73.2 & 71.4 & 99.7 & 91.6 & 73.1 & 70.1 \\
\hline
\end{tabular}

Note-Letter naming and position naming results are derived from this task.
Table 3

Summary of the Relevant Results: Room for

Improvement for Position Information $\left(1-P_{x c}\right)$, Benefits $(\Delta I)$, and Parameter 8 of Equation 2

\begin{tabular}{clccc}
\hline Experiment & \multicolumn{1}{c}{ Task } & $\left(1-P_{\mathrm{nc}}\right)$ & $\Delta I$ & 8 \\
\hline \multirow{2}{*}{1} & Letter naming & - & .032 & .70 \\
& Position naming & .046 & - & \\
& Letter-plus-position naming & .053 & .034 & .64 \\
2 & Letter-plus-position naming & .084 & .018 & .21 \\
\hline
\end{tabular}

termined. Percentages correct, averaged over days, are presented in Table 2.

An ANOVA of the correct letter identification scores showed that the effect of cuing was significant $[F(1,7)=$ $7.5, p<.05]$. The mean correct identification proportion was .732 with cuing and .714 without cuing. The position scores also revealed a significant cuing effect $[F(1,7)$ $=35.6, p<.01]$. Mean proportions correct for localization were .997 with cuing, and .916 without cuing. Scores for both letter and position correct again showed a significant cuing effect $[F(1,7)=22.0, p<.01]$. The proportions correct were .731 and .701 for cuing and nocuing conditions, respectively. Effects of days and blocks turned out to be not significant in either of the analyses.

In agreement with the model expressed in Equation 2, position information was not perfect; there is room for improvement in position information of .084. Moreover, this room for improvement is larger than the identity benefit brought by cuing of .018 . As was hypothesized, position information decreased by masking the onset of the letters. While identification performance was better than in Experiment 1, the proportion of trials on which position information was lacking increased from .046 in Experiment 1 to .084 in Experiment 2.

\section{GENERAL DISCUSSION}

According to the model expressed in Equation 2, identity benefits, $\Delta I$, brought by position cuing, are a fraction, $g$, of the room for improvement in terms of position information $\left(1-P_{n c}\right)$. For this model to be tenable, there must, of course, be a room for improvement in position information: $\left(1-P_{\mathrm{nc}}\right)>0$. Moreover, $\Delta I$ must be smaller than $\left(1-P_{\mathrm{nc}}\right)$, or, alternatively, parameter $g$ in Equation 2 should stand for a positive fraction: $0<g<1$.

Table 3 summarizes the results obtained. The column labeled $\left(1-P_{\mathrm{nc}}\right)$ shows that in all position-naming tasks there is a room for improvement: $\left(1-P_{\mathrm{nc}}\right)>0$. A comparison of the data in the columns labeled $\left(1-P_{\mathrm{nc}}\right)$ and $\Delta I$ reveals that $\Delta I$ is indeed smaller than $\left(1-P_{\mathrm{nc}}\right)$. And the column labeled $g$ shows that this parameter has theoretically acceptable values: $0<g<1$. In short, on the global level of averages, the outcome of the two experiments is completely consistent with the model summarized with Equation 2.

Having assessed the viability of Equation 2 as a model on a global level, it becomes worthwhile to investigate whether the equation also squares with the data in more detail. In this context, two issues deserve further attention. First, it is of interest to see whether also for each individual subject the room for improvement for position information $\left(1-P_{\mathrm{nc}}\right)$ is larger than the identity benefit, $\Delta I$, observed. Second, it is of relevance to see whether the identity benefits, $\Delta I$, can indeed be expressed as a linear function of the room for improvement in position information $\left(1-P_{\mathrm{nc}}\right)$.

For Experiment 1, inspection of the data under Letter and Position in Table 1 shows that for 2 out of the 6 subjects the inequality $\Delta I<$ $\left(1-P_{n c}\right)$ is violated. It is, however, important to realize that for this experiment the parameters entering in the inequality were obtained (1) in 
different conditions (the cue and the no-cue conditions), (2) with different tasks (a position-naming task and a letter-naming task), and (3) in separate blocks of trials (blocked presentation). It is therefore not unlikely that the violations observed resulted from irrelevant factors influencing the parameter estimates. This interpretation is supported by the results obtained in Experiment 2. For this experiment, the parameters entering the inequality were obtained also in different conditions (the cue and the no-cue conditions), but with the same type of task (a letter- plus position-naming task), and in the same series of stimulus presentations (mixed presentation). As the data in Table 2 show, in this experiment none of the 8 subjects showed a violation of the inequality.

For Experiments 1 and 2, the fitted linear functions relating $\Delta I$ to $\left(1-P_{\mathrm{nc}}\right)$ are, respectively,

$$
\begin{array}{ll}
\Delta I=.112\left(1-P_{\mathrm{nc}}\right)+.028 & (R=.171), \\
\Delta I=.193\left(1-P_{\mathrm{nc}}\right)+.002 & (R=.297) .
\end{array}
$$

While not really convincing, the functions are at least consistent with, and do not violate, the model expressed by Equation 2. Moreover, in interpreting the functions, two factors have to be taken into account.

The first factor concerns the estimate of $P_{\mathrm{nc}}$. It should be realized that, besides practical factors that limit our ability to estimate $P_{\mathrm{nc}}$, such as the subjects' guessing strategies and random factors, there is also in principle a problem with this parameter. The point is that the parameter of importance is not the proportion of trials without position information in the no-cue condition $\left(1-P_{n c}\right)$, but the proportion of trials on which the target provides no position information in the cue condition. However, we cannot estimate that proportion directly, and $\left(1-P_{n c}\right)$ has to be used as an estimate. But such an estimation procedure is not without problems. Because of differences in the visual situation (5 cues vs. 1 cue) and, possibly, in subject strategies (divided vs. focused attention), it is not clear whether $\left(1-P_{n c}\right)$ indeed offers an adequate estimate of the proportion of trials without position information in the cue condition.

The second factor concerns individual differences. Equation 2 states that, on the average, the benefits $\Delta I$ are a fraction, $g$, of the room for improvement for position information $\left(1-P_{\mathrm{nc}}\right)$. There is, however, sufficient reason to assume that each individual subject is characterized by his/her own unique parameter $g$. This intersubject variability is a source of error variation. The effect of this error variation on the fit of Equation 2 to the individual data points is inversely related to the size of variation in $\left(1-P_{n c}\right)$. As a result of the threshold procedure, used in the experiments reported here, the variation in $\left(1-P_{\mathrm{nc}}\right)$ is relatively small. Therefore the contribution of the error variation is relatively large. Consequently, only weak linear relations between $\Delta I$ and $\left(1-P_{\mathrm{nc}}\right)$ are to be expected.

One further piece of evidence suggests that Equation 2 is indeed correct as a model. This evidence consists of the data obtained in the no-cue conditions. The relevant data are presented in Table 4. Inspection of the two matrices and chi-square analyses indicate that there are strong dependencies between location processing and identity processing. (The chi-squares are 54.2 and 716.5 for Experiments 1 and 2, respectively; $d f=1, p<.001$.) Because position processing is appreciably better than identity processing, the correct identification data in the no-cue condition can be expressed in terms of conditional probabilities, $I+\mid L+$ and $I+\mid L-$, where $I+$ stands for correct identification and $L+$ and $L-$ for correct and incorrect localization, respectively. For Experiment 1, $I+\mid L+$ equals .643 and $I+\mid L-$ equals .232 . For Experiment 2 , these values are .765 and .165 , respectively. These figures show that the probability of a correct identification given an incorrect localization is very
Table 4

Results Obtained in the No-Cue Conditions of the Letter- Plus Position-Naming Tasks in Experiments 1 and 2

\begin{tabular}{ccrr}
\hline & & \multicolumn{2}{c}{ Identity } \\
\cline { 2 - 4 } Experiment & Position & $I+$ & \multicolumn{1}{c}{$I-$} \\
\hline 1 & $P+$ & 874 & 485 \\
& $P-$ & 19 & 62 \\
2 & $P+$ & 3,589 & 1,101 \\
& $P-$ & 67 & 363 \\
\hline
\end{tabular}

Note $-P+=$ position correct. $P-=$ position incorrect. $I+=$ identity correct. $I-=$ identity incorrect. Table entries are the observed frequencies for each $P \times I$ combination.

low. In fact, these probabilities are quite close to chance probability (.125). So it appears that also in the no-cue condition position information is (close to) essential for a correct identification response.

The fact that in the no-cue condition location information is (close to) essential for a correct identification response supports the view that Equation 2 as a model is indeed correct for the following reason. Suppose that it does not matter whether there is position information or notthat is, suppose that $I+\mid L+$ equals $I+\mid L-$. Then it is very hard to see how irrelevant position information, brought by a location cue, could produce benefits. Benefits are to be expected, however, if position information matters and is (close to) essential for a correct identification response. Then the cue is capable of bringing the position information when the target letter was not capable of bringing that information itself. So, just because in the no-cue condition position information that is (close to) essential for a correct identification response is sometimes lacking, the model embodied in Equation 2 is the most parsimonious way to account for the benefits brought by position cuing.

\section{REFERENCES}

Egly, R., \& НомA, D. (1984). Sensitization of the visual field. Journal of Experimental Psychology: Human Perception \& Performance, 10, 778-793.

Grindley, G. C., \& Townsend, V. (1968). Voluntary attention in peripheral vision and its effects on acuity and differential thresholds. Quarterly Journal of Experimental Psychology, 20, 11-19.

VAN DER HEIJDEN, A. H. C. (1991). Selective attention in vision. London, New York: Routledge.

Van der Heijden, A. H. C., NeerincX, M., \& Wolters, G. (1989). Location cuing benefits with exposure duration variations. Bulletin of the Psychonomic Society, 27, 35-38.

VAN der Heijden, A. H. C., SChreuder, R., \& Wolters, G. (1985). Enhancing single-item recognition accuracy by cueing spatial locations in vision. Quarterly Journal of Experimental Psychology, 37A, 427-434.

Van der Heidden, A. H. C., Wolters, G., Groep, J. C., \& HAGENAAR, R. (1987). Single-letter recognition accuracy benefits from advance cuing of location. Perception \& Psychophysics, 42, 503-509.

YANTIS, S., \& JoNidES, J. (1984). Abrupt visual onsets and selective attention: Evidence from visual search. Journal of Experimental Psychology: Human Perception \& Performance, 10, 601-621.

(Manuscript received July 5, 1991.) 